\title{
Nonlinear Fault Estimation Methods for Semi-Active Suspension Dampers *
}

\author{
Marcelo M. Morato* Olivier Sename ${ }^{\dagger}$ Luc Dugard $^{\dagger}$ \\ * Grupo de Pesquisa em Energias Renováveis (GPER), Departamento \\ de Automação e Sistemas (DAS), Universidade Federal de Santa \\ Catarina (UFSC), Florianópolis-SC, Brazil, \\ (marcelomnzm@gmail.com). \\ ${ }^{\dagger}$ Univ. Grenoble Alpes, CNRS, Grenoble INP, GIPSA-lab, 38000 \\ Grenoble, France. \\ (\{olivier.sename,luc.dugard\}@gipsa-lab.fr)
}

\begin{abstract}
The design of Fault Estimation methods for Electro-Rheological dampers of automotive suspension systems is considered in this paper. Multiplicative loss of effectiveness actuator factors are considered, which are estimated by two distinct approaches: i) an $L P V$ asymptotic polytopic observer; and ii) a sliding-mode fault reconstruction technique. While the first uses a mixed $H_{2} / H_{\infty}$ performance objective to reduced disturbance effects, the later has a simple synthesis procedure by using a fixed input distribution matrix and ensuring sliding motion. Results are discussed through simulation, demonstrating the truthfulness and capability of these tools to estimate Electro-Rheological damper faults.

Resumo: Métodos de Estimativa de Falhas para amortecedores Eletroreológicos de suspensões automotivas são considerados neste trabalho. Falhas de perda de eficiência na atuação são consideradas, sendo estimadas por duas abordagens distintas: i) observador $L P V$ politópico e assintótico; e ii) reconstrução de falhas baseada em modos deslizantes. Enquanto a primeira visa dirimir o efeito das perturbações ao minimizar um objetivo de desempenho $H_{2} / H_{\infty}$, a segunda possui uma síntese simples por uso de uma matriz fixa de distribuição que garante o movimento deslizante. Resultados são discutidos através de simulação, demonstrando a capacidade dos métodos de estimar falhas em amortecedores automotivos eletroreológicos.
\end{abstract}

Keywords: Fault Estimation; LPV Observer; Sliding Mode; Semi-Active Suspensions; ER Dampers.

Palavras-chaves: Estimativa de Falhas; Observador LPV; Modos Deslizantes; Suspensões Semi-Ativas; Amortecedores ER.

\section{INTRODUCTION}

Automotive engineering has recently come to know the use of modern active safety and comfort features, such as controlled suspension systems. Semi-Active $(S A)$ suspension systems are efficient, while being less expensive and energy-consuming than purely active ones. This type of suspension can be sighted on new top-cars and in a good deal of academic and industrial research, see Savaresi et al. (2010), being able to enhance ride performance (i.e. passenger comfort) if smoothly controlled. Anyhow, modern vehicle suspensions are subject to possible faults, failures and component malfunctions; for instance, the damping fluid might leak, providing smaller damping. These events imply performance degradation or even loss of control.

Attention has been considerably given to Fault Tolerant Control $(F T C)$ schemes, that offer increased process availability by avoiding breakdowns from simple faults, see Blanke et al. (1997). Active FTC requires online Fault De-

* This work has been supported by CAPES BRAFITEC ECoSud and by the Investissements d'avenir LabEx PERSYVAL-Lab $(A N R-11-L A B X-0025-01)$. tection and Isolation/Fault Estimation $(F D I / F E)$ strategies, so that the control scheme knows real-time information about the state of the plant (faulty/healthy) to compute, if necessary, an adequate reconfiguration mechanism. Literature shows that the modular design (FDI/FE and FTC designed separately) is more flexible for practical applications and easier to test and implement, see Jiang and $\mathrm{Yu}(2012)$.

Even though many studies have been concerned with FDI, fewer have considered FE methods. Among them, some are: the adaptive fault estimation method for nonlinear plants, see Zhang et al. (2012), the robust observer in Gao and Ding (2007) and the Moving-Horizon Estimation schemes of Gatzke and Doyle Iii (2002).

From the 2000's, some extensions of the linear FE methods to $(L P V)$ systems ${ }^{1}$ have been developed. $L P V \quad F E$ schemes are able to autonomously adjust and schedule observer/detection-filter gains, which is a suitable tradeoff between full scaled nonlinear designs and LTI methods.

\footnotetext{
1 LPV systems can be understood as a range of systems with known, bounded parameter variations.
} 
Some recent $L P V F E$ works are: Chen et al. (2016) show their application to an aerospace benchmark, while Rotondo et al. (2016) develops switched observers for discrete $L P V$ plants.

\subsection{FE for ER Automotive Dampers}

This article focuses on the use of $F E$ methods for $(S A)$ Electro-Rheological $(E R)$ dampers $^{2}$, which can fail due to oil leakages, physical deformation or even the presence of air in the ER damping fluid. While there are controloriented works for such $E R$ dampers, the study of faults, failures and monitoring is quite novel, albeit being of high interest for real cars. Only some few works have dealt with the issue of faulty $(E R)$ dampers (and only considering a global actuator fault, instead of a loss of effectiveness), mainly in FTC problems, see Sename et al. (2013), TudónMartínez et al. (2013).

Estimating their loss of effectiveness is of great interest for safety and FTC strategy. Moreover, the focus here is to compare a simple-to-implement $L P V$ observer-based $F E$ method presented by authors in Morato et al. (2019) with the nonlinear sliding-mode technique as proposed by Edwards et al. (2000) (which also has a simple design procedure and a wide range of applicability).

Regarding the $E R$ damper $F E$ problem, very few recent works concern the modelling of loss of effectiveness fault factors, as done in Hernández-Alcántara et al. (2016); Nguyen et al. (2016). However, such estimation methods are quite complex to implement in real embedded computers. Therefore, more practical methods must be investigated, such as those based on $L P V$ or sliding-mode observers. The objective of this paper is to compare these two kinds ( $L P V$, sliding-mode) of methods. The contributions, thereof, are:

(1) An $L P V F E$ method is developed, considering a polytopic LPV Extended-Observer design, with mixed $H_{2} / H_{\infty}$ performance problem (Sec. 3 );

(2) A sliding-mode technique for $F E$ is detailed, for comparison purposes (Sec. 4);

(3) Simulation results are presented, highlighting the accuracy/success of both methods and concluding on their advantages/disadvantages (Sec. 5).

\section{ER SA SUSPENSION SYSTEM}

A $S A$ vehicle system $^{3}$ with four Electro-Rheological dampers is studied. The presented vehicle, tire, spring and damper models are well known in literature and readers are invited to refer to Poussot-Vassal et al. (2012) for more details. The use of semi-active suspension systems provides a good balance between costs and performance requirements as explained, for instance, in Savaresi et al. (2010).

\footnotetext{
2 Semi-Active suspensions are thoroughly detailed in Sec. 2.

3 An automotive suspension system comprises, basically, two components: a spring and a damping structure. These components have to work together to maintain the tire's contact to the ground. The goal of the damping structure is to reduce the effect of travelling upon a rough road by absorbing shock and helping with driving performance, ensuring a smoother and safer ride (comfort).
}

The dynamics are usually described by the Quarter-ofVehicle $(Q \circ V)$ model, as details Savaresi et al. (2010), comprising the vertical motion of the chassis $\left(z_{s}\right)$ and of the axle $\left(z_{u s}\right)$. A full nonlinear vertical model of the vehicle is used as a simulation tool, later on. The tire forces $\left(F_{t_{i j}}\right)$ are considered as proportional to the wheel deflection, as gives Eq. (1), where $k_{t_{i j}}$ represents the stiffness coefficients of the tires and $z_{r_{i j}}$ stand for the road profile disturbances. Each vertical suspension force, represented by $F_{s_{i j}}$, is modelled by a spring and a damper with passive and semiactive parts, as describes Eq. (2), where $u_{i j}$, the control input, satisfies some dissipativity constraints. Remark that $z_{d e f_{i j}}=z_{s_{i j}}-z_{u s_{i j}}$ stands for the suspension deflection ${ }^{4}$.

$$
\begin{aligned}
& F_{t_{i j}}(t)=k_{t_{i j}} \overbrace{\left(z_{u s_{i j}}(t)-z_{r_{i j}}(t)\right)}^{\text {Wheel deflection }} \\
& F_{s_{i j}}(t)=\underbrace{\text { Nominal damping }}_{\text {Spring }} \\
&+\overbrace{c_{0_{i j}} \dot{z}_{\text {def } f_{i j}}(t)}^{u_{i j}(t)} \\
& \underbrace{}_{\text {Control input }}(t)
\end{aligned}
$$

Possible faults of $S A$ dampers may occur due to internal oil leakages, physical deformation, due to electrical problems on the amplifiers used to control the dampers, or even to the presence of air in the damping fluid. In practice, they convert into an energetic loss of effectiveness. This fault representation has been firstly presented in Sename et al. (2013) and Tudón-Martínez et al. (2013) and later used in Hernández-Alcántara et al. (2016), which introduced a solid framework for the modelling of faults. Henceforth, faults are represented by a multiplicative factor $\alpha$ upon each controlled damper force $u$.

Remark 1. Generally speaking, the real actuation upon the suspension system, given by $u_{f}(t)$, depends proportionally to the damper force $u(t)$. In a faultless situation, $\alpha=1$ and when the damper achieves an uncontrollable condition, i.e. with only passive $\left(c_{0}\right)$ behaviour, $\alpha=0$. Thus: $\alpha(t) \in[0,1]$, with $u_{f}(t)=\alpha(t) u(t)$.

The state-space representation of the (faulty) QoV model is found by taking the system states as Eq. (4) and the disturbances as the road profile given in Eq. (5). The outputs are the suspension deflection, chassis acceleration and deflection velocity, as depicts Eq. (6), which are easily measurable with on-board vehicle sensors (acceleration/inertial units and deflection sensor), widely present in modern cars. Thus, for each corner:

$$
\left.\begin{array}{l}
\dot{x}(t)=A x(t)+B_{1} w(t)+B_{2} \alpha(t) u(t) \\
y(t)=C x(t)+D_{1} w(t)+D_{2} \alpha(t)(t)
\end{array}\right\}
$$

with constant matrices $A$ to $D_{2}$.

$$
\begin{aligned}
& x(t)=\left[\begin{array}{llll}
z_{s}(t) & \dot{z}_{s}(t) & z_{u s}(t) & \dot{z}_{u s}(t)
\end{array}\right]^{T} \\
& w(t)=z_{r}(t) \\
& y(t)=\left[\begin{array}{lll}
z_{\text {def }}(t) & \ddot{z}_{s}(t) \dot{z}_{\text {def }}(t)
\end{array}\right]^{T}
\end{aligned}
$$

Assumption 1. In the sequel, it is assumed that some model (portion) of the road profile disturbance is known. This can be expressed mathematically by:

\footnotetext{
${ }^{4}$ Subscripts $i-j$ stand for (front/rear)-(left/right) corners.
} 


$$
\begin{gathered}
w(t)=\overbrace{w_{\mathrm{m}}(t)}^{\text {known model }}+\overbrace{\delta w(t)}^{\text {unknown }}, \\
\dot{w}_{\mathrm{m}}(t)=A_{m w} w_{\mathrm{m}}(t)+\overbrace{\nu(t)}^{\text {noise }} .
\end{gathered}
$$

Such kind of description allows to consider several types of road profiles; $\delta w(t)$ allows to include some modelling uncertainties. The knowledge of a road profile model can be achieved on-line either using additional components (as frontal cameras) or using some algorithms, such as the adaptive road profile estimators proposed by TudónMartínez et al. (2015) and Doumiati et al. (2017).

Assumption 2. It is also assumed that the measured outputs $y(t)$ are as well subject to some additive noise $\nu(t)$. This is typical in any real instrumented plant; so, one has:

$$
y(t)=C x(t)+D_{1} w(t)+D_{2} u_{f}(t)+D_{\nu} \nu(t),
$$

where $D_{\nu}$ is a noise distribution matrix.

Assumption 3. Last but not least, it is also assumed (and reaffirmed) that the fault factor $\alpha(t)$ is slowly-varying, i.e. $\dot{\alpha}(t)$ is taken as 0 .

\subsection{Model Parameters}

A real mechatronic test-bench is considered as the model for validation of the $F E$ strategies. This testbed is the INOVE Soben-Car, a $\frac{1}{5}$-scaled vehicle, which allows testing several configurations and use-cases (refer to full details in www.gipsa-lab.fr/projet/inove). In Table 1, the numerical values for each of the parameters of this vehicle testbed are given, considering the front-right corner.

Table 1. Vehicle Model Parameters

\begin{tabular}{ccc}
\hline Parameter & Value & Unit \\
\hline$m_{s}$ & 2.27 & $\mathrm{~kg}$ \\
$m_{u s}$ & 0.25 & $\mathrm{~kg}$ \\
\hline$k_{t}$ & 12269.81 & $\mathrm{~N} / \mathrm{m}$ \\
\hline$k_{s}$ & 1396.80 & $\mathrm{~N} / \mathrm{m}$ \\
\hline
\end{tabular}

\section{LPV FE OBSERVER METHOD}

The LPV FE Observer method from Morato et al. (2019) is now described. Equations (7), (8) and (9) are coupled and the extended state-space representation arises:

$$
\begin{aligned}
& \overbrace{\left[\begin{array}{c}
\dot{x}(t) \\
\dot{\alpha}(t) \\
\dot{w}_{m}(t)
\end{array}\right]}^{\dot{x_{a}(t)}}=\overbrace{\left[\begin{array}{ccc}
A & B_{2} \rho(t) & B_{1} \\
0 & 0 & 0 \\
0 & 0 & A_{m w}
\end{array}\right]}^{A_{a}(\rho)} \overbrace{\left[\begin{array}{c}
x(t) \\
\alpha(t) \\
w_{m}(t)
\end{array}\right]}^{x_{a}(t)} \\
& +\overbrace{\left[\begin{array}{c}
B_{1} \\
0 \\
0
\end{array}\right]}^{B_{w}} \delta w(t)+\overbrace{\left[\begin{array}{l}
0 \\
0 \\
\mathbb{I}
\end{array}\right]}^{B_{\nu}} \nu(t), \\
& y(t)=\underbrace{\left[\begin{array}{lll}
C & D_{2} \rho(t) & D_{1}
\end{array}\right]}_{C_{a}(\rho)} x_{a}(t) \\
& +D_{1} \delta w(t)+D_{\nu} \nu(t),
\end{aligned}
$$

where

$$
\rho(t)=u(t) .
$$

Due to physical saturation constraints of $S A E R$ dampers, $\rho$ is naturally bounded, since:

$$
u(t) \in \mathcal{U}_{\text {sat }}=\left\{u_{\min } \leq u(t) \leq u_{\max }\right\} .
$$

Herein, one defines the scheduling parameter's polytope $\mathcal{P}$, whose vertices are given by the boundaries of $\mathcal{U}_{\text {sat }}$. It is important to notice that the matrices $\mathbf{A}_{a}$ and $\mathbf{C}_{a}$ are affine in $u(t)$, which ensures that a polytopic approach can be used to represent the $L P V$ system given by Eq. (10).

Since the faulty system is represented via an augmented framework with states $x_{a}(t)$, the estimation of $\alpha(t)$ is directly achieved by tracking these augmented states. As explained in Rodrigues et al. (2015), an asymptotical $L P V$ observer to track the states $x_{a}(t)$ is:

$$
\begin{aligned}
\dot{\hat{x}}_{a}(t) & =\mathbf{A}_{a}(\rho) \hat{x}_{a}(t)+\mathbf{L}(\rho)\left[y(t)-\mathbf{C}_{a}(\rho) \hat{x}_{a}(t)\right], \\
\hat{\alpha}(t) & =\underbrace{\left[\begin{array}{lll}
0_{\operatorname{size}(x)} & \mathbb{I}_{\operatorname{size}(\alpha)} & 0_{\operatorname{size}(w)}
\end{array}\right]}_{\mathbf{E}} \hat{x}_{a}(t),
\end{aligned}
$$

where $\hat{x}_{a}(t)$ and $\hat{\alpha}(t)$ stand, respectively, for the estimation of the augmented states and the fault term.

The dynamics of the estimation error $\left(e(t)=x_{a}(t)-\hat{x}_{a}(t)\right)$ and fault estimation error $\left(e_{\alpha}(t)=\alpha(t)-\hat{\alpha}(t)\right)$ are given by:

$$
\begin{aligned}
\dot{e}(t)= & {\left[\mathbf{A}_{a}(\rho)-\mathbf{L}(\rho) \mathbf{C}_{a}(\rho)\right] e(t) } \\
& +\left(\mathbf{B}_{w}-\mathbf{L}(\rho) \mathbf{D}_{1}\right) \delta w(t) \\
& +\left(\mathbf{B}_{\nu}-\mathbf{L}(\rho) \mathbf{D}_{\nu}\right) \nu(t), \\
e_{\alpha}= & \mathbf{E} e(t) .
\end{aligned}
$$

As seen in Equation (14), the stability of the estimation error depends on the choice of the gain matrix $\mathbf{L}(\rho)$. Therefore, according Morato et al. (2019), a mixed $H_{2} / H_{\infty}$ sensitivity criterion is followed, i.e. find a gain matrix $\mathbf{L}(\rho)$, affine in the scheduling parameter $\rho$ s.t. dynamics (14)-(15) are exponentially stable when $\nu(t)$ and $\delta w(t)$ are null, and, s.t. the following costs are minimized under $\left.e(t)\right|_{t=0}=$ $0:\left\|\frac{e_{\alpha}}{\nu}\right\|_{2} \leq \gamma_{H_{2}}$ for $\delta w(t) \equiv 0 ;$ and $\left\|\frac{e_{\alpha}}{\delta w}\right\|_{\infty} \leq \gamma_{H_{\infty}}$ for $\nu(t) \equiv 0$. The solution to this mixed $H_{2} / H_{\infty} L P V$ observer design is given by a set of $L M I \mathrm{~s}$, see Lemma 1 in Morato et al. (2019). This mixed criterion is used because it can guarantee insensitivity towards noise (in the $H_{2}$ sense) together with insensitivity face road (load) disturbances (in the $H_{\infty}$ sense).

Remark 2. As there is only one scheduling parameter $\rho$, this method is concerned only in solving the given $L M I$ problem at $\rho=u_{\max }$, finding $\mathbf{L}_{\max }$, and at $\rho=u_{\min }$, finding $\mathbf{L}_{\text {min }}$. Then, the gain matrix $\mathbf{L}(\rho)$ is taken as affine in $\rho$, and guarantees the stability of the estimation error dynamics, just as:

$$
\begin{aligned}
L(\rho) & =\left(\frac{\rho_{\max }-\rho}{\rho_{\max }-\rho_{\min }}\right) L_{\min } \\
& +\left(\frac{\rho-\rho_{\min }}{\rho_{\max }-\rho_{\min }}\right) L_{\max } .
\end{aligned}
$$

\section{SLIDING-MODE FE METHOD}

Now, consider the use of a (nonlinear) sliding-mode observer to determine the value of the loss of effectiveness on the ER damper, $\alpha$. This method is adapted from Edwards et al. (2000), for comparison purposes with the previous one. 
Consider that the system states can be split in two parts: those states that are insensible to faults $\left(x_{1}(t)=\right.$ $\left.\left[z_{s}(t) z_{u s}(t)\right]\right)$ and those which suffer from fault degradation $\left(x_{2}(t)=\left[\dot{z}_{s}(t) \dot{z}_{u s}(t)\right]\right)$. Using the $Q o V$ model, a new representation arises:

$$
\begin{aligned}
\dot{x_{1}}(t) & =\mathcal{A}_{11} x_{1}(t)+\mathcal{A}_{12} x_{2}(t), \\
\dot{x_{2}}(t) & =\mathcal{A}_{11} x_{1}(t)+\mathcal{A}_{12} x_{2}(t) \\
& +\mathcal{B}_{1_{2}} w(t)+\mathcal{B}_{2_{2}} u(t)+\mathcal{D}_{2}(\rho) f_{i}(t),
\end{aligned}
$$

where $\mathcal{A} \in \mathbb{R}^{n \times n}$ is a linear transformation on states $\left[x_{1}^{T}(t) x_{2}^{T}(t)\right]^{T}=T_{x} x(t)$, this is $\mathcal{A}=T_{x} A T_{x}^{-1}, \mathcal{B}_{1}=$ $T_{x} B_{1}, \mathcal{B}_{2}=T_{x} B_{2}$, and $\mathcal{D}(\rho)$ represents the fault distribution matrix. Note that, with this formulation, the road disturbance $w(t)$ and control signal $u(t)$ only influence $x_{2}(t)$. Moreover, for this method, the fault term $f_{i}(t)$ derives from $f_{i}(t)=(\alpha(t)-1) u(t)$.

It is assumed that $\mathcal{D}(\rho)$ can always be decomposed into fixed and a varying component, $\mathcal{D}(\rho)=D E(\rho)$, for $D \in$ $\mathbb{R}^{n \times q}$ and $E(\rho) \in \mathbb{R}^{q \times q}$. It is important to remark that $r_{1}(\rho)$ and $\psi: \mathbb{R}_{+} \times \mathbb{R}^{p} \times \mathbb{R}^{d} \rightarrow \mathbb{R}_{+}$are known, well-defined functions. Taking $\rho=u(t)$, it is implied that $D=\mathcal{B}_{2}$ and $E(\rho)=\rho \mathbb{I}$. Then, consider $f_{v}(t, \rho): \mathbb{R}_{+} \times \mathbb{R}^{d} \rightarrow \mathbb{R}^{q}$ a function that represents "virtual faults" upon the dampers. Moreover, these "virtual faults" are assumed to obey:

$$
\left\|f_{v}(t, \rho)\right\|<r_{1}(\rho)\|u(t)\|+\psi(t, y, \rho) .
$$

The determinant $\operatorname{det}(E(\rho))$ is taken as $\operatorname{det}(E(\rho)) \neq 0$ and, for this, the actual fault factor $\alpha$ will be able to be retrieved from the estimative $f_{v}(t, \rho)$. The functions $r_{1}(\rho)$ and $\psi(t, y, \rho)$ are defined so to constitute the upper bound on the worst of a loss of effectiveness fault on the system dynamics (this is, for $\alpha=0$ ), as shows Eq. (19).

The fault observer for this second method little modifies from Eq. (13), but now gives:

$$
\begin{aligned}
\dot{\dot{x}_{1}}(t) & =\mathcal{A}_{11} \hat{x_{1}}(t)+\mathcal{A}_{12} \hat{x_{2}}(t) \\
& -G_{L_{1}} e_{y}(t)-G_{N_{1}} \varrho_{1}(t), \\
\dot{x_{2}}(t) & =\mathcal{A}_{21} \hat{x_{1}}(t)+\mathcal{A}_{22} \hat{x_{2}}(t) \\
& -G_{L_{2}} e_{y}(t)-G_{N_{2}} \varrho_{2}(t) \\
& +\mathcal{B}_{1_{2}} \hat{w}(t)+\mathcal{B}_{2_{2}} u(t) .
\end{aligned}
$$

With this method, instead of defining the gain matrix $L(\cdot)$, one has to define the gain matrices $G_{L}$ and $G_{N}$ and the auxiliary input $\varrho(t)$. These are so that a sliding motion is induced and the output estimation error $e_{y}(t)=x_{2}(t)-$ $\hat{x}_{2}(t)=\mathcal{C}(x(t)-\hat{x}(t))$ goes to zero in finite time. Once this is true, a first order sliding mode is obtained on surface $\mathcal{S}$ :

$$
\mathcal{S}=\left\{e \in \mathbb{R}^{n}: \mathcal{C} e=0\right\}
$$

Remark that the observer from Eqs. (20)-(21) is purely a state-observer and does not consider the augmented states as in the previous approach. The information on the disturbance $\hat{w}(t)$ is generated by a parallel scheme, according to Assumption 1. Thus, the estimation dynamics are:

$$
\begin{aligned}
\dot{e_{1}}(t) & =\mathcal{A}_{11} e_{1}(t)+\mathcal{A}_{12} e_{2}(t) \\
& +G_{L_{1}} \cdot e_{y}(t)+G_{N_{1}} \varrho_{1}(t), \\
\dot{e_{2}}(t) & =\mathcal{A}_{21} e_{1}(t)+\mathcal{A}_{22} e_{2}(t) \\
& +G_{L_{2}} e_{y}(t)+G_{N_{2}} \varrho_{2}(t)+D_{2} f_{v}(t, \rho) .
\end{aligned}
$$

As proposed by Drakunov and Utkin (1995), the design tools that induce the sliding mode on the surface $\mathcal{S}$ are attained such as adequate, fast stabilization of the error dynamics, Eqs. (23)-(24), are yielded. Therefore, one takes:

$$
\begin{aligned}
G_{L_{1}} & =-\mathcal{A}_{12}, \\
G_{N_{1}} & =\mathcal{L}=\left[\begin{array}{cc}
-\lambda_{1} & 0 \\
0 & -\lambda_{2}
\end{array}\right], \\
\lambda_{1} & \geq \lambda_{2}>0, \\
\varrho_{1}(t) & =\breve{e}_{1}(t),
\end{aligned}
$$

which means $\mathcal{L}$ is chosen with fast-enough eigenvalues $\lambda_{j}$ and $\breve{e}_{1}(t)$ represents an (open-loop) estimative of the prediction error $e_{1}$. Taking, for simplicity, $\breve{e}_{1}(t)=e_{1}(t)$, one finds $\dot{e_{1}}(t)=\mathcal{L} e_{1}(t)$, which is Lyapunov-stable (and converges to zero in finite time).

The degrees-of-freedom on $\dot{e_{2}}(t)$ are taken as follows: expand Eq. (24), considering that $w(t)-\hat{w}(t) \rightarrow \epsilon_{w}$, $e_{1}(t) \rightarrow \epsilon_{e}$ :

$$
\begin{aligned}
G_{L_{2}} & =\left(\mathcal{A}_{22}^{\text {stab }}-\mathcal{A}_{22}\right), \\
\varrho_{2}(t) & =\mu \| D_{2}|| \operatorname{sign}\left(e_{y}(t)\right),
\end{aligned}
$$

where $\varrho_{2}(t)$ is the sliding-mode injection term ${ }^{5}$ and $\mu$ is an appropriate scalar s.t $\left\|\mathcal{B}_{1_{2}} \epsilon_{w}\right\|+\left\|\mathcal{A}_{12} \epsilon_{e}\right\|+\left\|f_{v}(t, \rho)\right\|<\mu$. Moreover, $G_{N_{2}}$ is a Lyapunov matrix for $\mathcal{A}_{22}^{\text {stab }}$. With these parameters, one obtains:

$$
\begin{aligned}
\mathbb{I}_{2} & =G_{N_{2}} \mathcal{A}_{22}^{\text {stab }}+\left(\mathcal{A}_{22}^{\text {stab }}\right)^{T} G_{N_{2}}, \\
\dot{e_{2}}(t) & =\mathcal{A}_{22}^{\text {stab }} e_{2}(t)+\underbrace{G_{N_{2}} \varrho_{2}(t)}_{\text {Sliding-Mode Term }} \\
& +D_{2} f_{v}(t, \rho)+\underbrace{\left(\mathcal{A}_{12} \epsilon_{e}+\mathcal{B}_{1_{2}} \epsilon_{w}\right)}_{\text {ideally }=0} .
\end{aligned}
$$

Finally, fault information is retrieved as follows: it is assumed that the sliding-mode observer has been well designed and that the sliding motion on the surface $\mathcal{S}$ has been established; then, during the sliding motion, it is true that $e_{y}(t)=0$ and $\frac{d e_{y}(t)}{d t}=0$, considering the assumptions presented beforehand. Therefore $G_{N_{2}} \nu_{2}^{\text {sliding }}(t) \rightarrow$ $-D_{2} f_{v}(t, \rho)$ and:

$$
\begin{aligned}
\dot{e_{2}}(t) & =\overbrace{\left[\mathcal{A}_{21} e_{1}(t)+\mathcal{B}_{1_{2}}(\hat{w}(t)-w(t))\right]}^{\text {stable convergence }} \\
& +\mathcal{A}_{22}^{\text {stab }} e_{2}(t)+G_{N_{2}} \varrho_{2}(t)+D_{2} f_{v}(t, \rho) .
\end{aligned}
$$

Function $\varrho_{2}(t)$ is discontinuous, but can replaced by a continuous approximation, considering a suitable (smallenough) choice of $\sigma$. This is, for every component $k$, with $t=k T_{s}$ and $T_{s}=5 \mathrm{~ms}$, of $\varrho_{2}(t)$, one takes:

\footnotetext{
5 A complete analysis on the sliding-mode injection term $\nu_{2}(t)$ is exposed on Moreno and Osorio (2008), with results on stability and proofs that the output error will converge to zero in finite time, considering the suitable choice of $G_{N_{2}}$.
} 


$$
\left(\nu_{2}^{\text {sliding }}\right)_{k}(t) \approx \mu\left\|D_{2}\right\| \frac{e_{y_{k}}(t)}{\left\|e_{y_{k}}(t)\right\|+\sigma}
$$

Finally, the fault information is retrieved as goes:

- Compute the approximate $\varrho_{2}^{\text {sliding }}(t)=\operatorname{col}\left\{\left(\varrho_{2}^{\text {sliding }}\right)_{k}(t)\right\}$

- Retrieve $\hat{f}_{v}(t, \rho)=-\breve{\mathcal{D}}_{2}{ }^{\star}\left(G_{N_{2}} \varrho_{2}^{\text {sliding }}\right)$, for ${\breve{\mathcal{D}_{2}}}^{\star}$ being the pseudo-inverse of ${\breve{\mathcal{D}_{2}}}_{2}$;

- Reconstruct $f_{i}(t)$ as of $\hat{f}_{i}(t)=E^{-1}(\rho) \hat{f}_{v}(t, \rho)$;

- Take $\hat{\alpha}=1+\hat{f}_{i}$.

\section{SIMULATION RESULTS}

The main contribution of this paper is now presented, which is the comparison between both $F E$ methods for $E R$ damper faults on $S A$ suspension systems.

So to provide realistic results, the simulation considers a full nonlinear vehicle model, as described in Poussot-Vassal et al. (2011),Fergani et al. (2016). This model includes nonlinear suspension forces and has been validated with a real car. In order to mimic measurement noise, a highfrequency signal $(\nu(t))$ is added to each component of $y(t)$. Moreover, a sinusoidal $15 \mathrm{~mm}$ road profile $w(t)$ that represents a series of bumps for a vehicle running on a dry road at constant speed, is used, see Doumiati et al. (2017). The suspension $E R$ damper is initially fault-less $(\alpha(0)=1)$.

For this, a simple single-step scenario is taken, wherein the loss of effectiveness fault $\alpha(t)$ decreases abruptly, at $t=25 \mathrm{~s}$, to 0.85 . Figure 1 shows the expected (faultless) damper force $u(t)=F_{E R}(t)$ compared to the faulty $u_{f}(t)=\alpha(t) F_{E R}(t)$, according to the measured outputs $y(t)$, which are given in Figure 2. Note that the ER damper force comes from a Force Control System that provides a control action aiming to enhance the comfort of passengers.

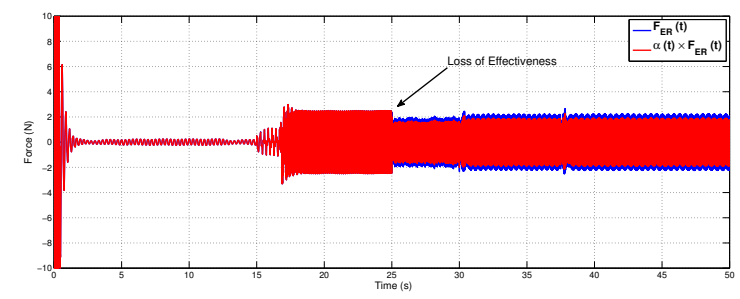

Figure 1. Faulty and Fault-Free ER Damper Force

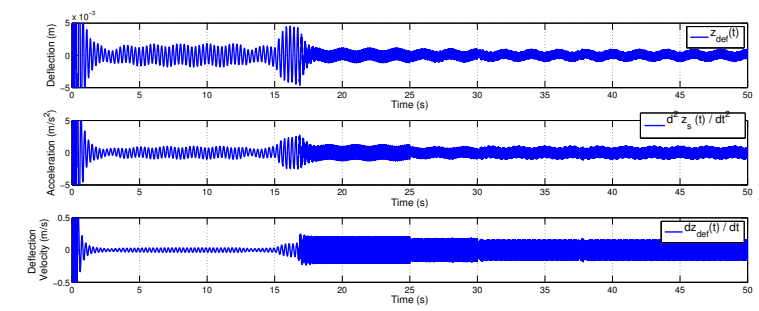

Figure 2. Measured Outputs and Deflection Velocity

Then, the estimation of $\hat{\alpha}(t)$ by both approaches is given in Figure 3, compared to the actual value of $\alpha(t)$. It can be seen, therefore, that compared with the sliding-mode fault reconstruction approach (discussed in Edwards et al. (2000), Xiao et al. (2012) and Hamayun et al. (2016)), the polytopic $L P V$ observer from Morato et al. (2019) yields far more interesting results: even though the sliding) \} mode method is very fast and can conclude if fault have happened in a matter of few milliseconds, it is not able to precisely quantify how much loss does the $E R$ damper in fact present, while the $L P V$ observer can very well quantify this loss of $25 \%$ on the actuation.

For fault tolerance goals, for instance, accurate fault estimation is necessary, such that re-configure the control laws can be found in such way that driving performances of the vehicle are maintained. With the sliding-mode approach, this would not be possible, but with $L P V$ observer method direct.

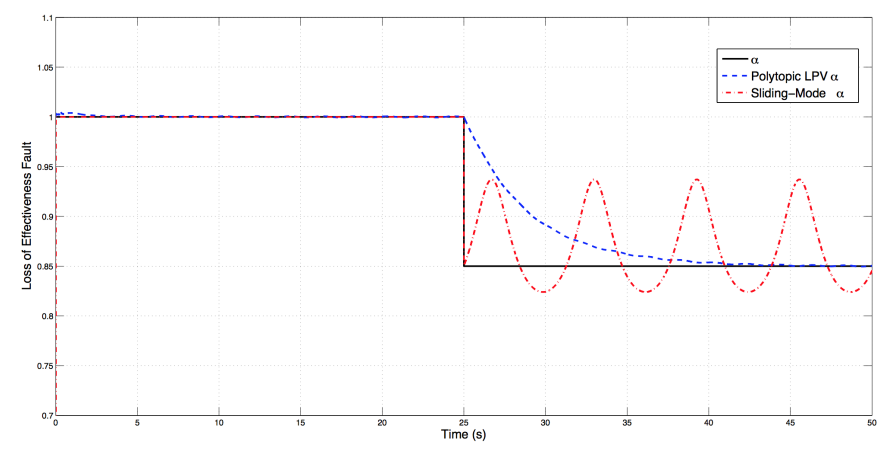

Figure 3. Simulation of Fault Estimation Comparison to Sliding-Mode Approach

\section{CONCLUSIONS}

This paper compared two nonlinear methods for fault estimation in Electro-Rheological automotive dampers: an $L P V$-based observer approach and a sliding-mode fault reconstruction one. With realistic simulations, it is shown that the $L P V$ scheme is able to efficiently estimate the loss of effectiveness (state of health) of the $E R$ damper, thanks to mixed $H_{2} / H_{\infty}$ performance objectives, while the sliding-mode scheme (albeit being very fast) can only conclude if the damper is in a faulty state, but cannot quantify the loss with precision.

In terms of implementation cost, let it be stressed that:

- The considered sensors for both methods are the ones used for control purposes of the vertical car dynamical behaviour, namely the suspension deflection and the sprung mass acceleration (whereas the deflection velocity is computed numerically). Therefore, no additional sensors on the $E R$ damper itself are needed, only the on-board vehicle sensors. As a decoupled $Q o V$ model is used, both $F E$ approaches can be designed individually for each corner of the vehicle, which provides simplicity and a straightforward implementation that could be done on simple microcontrollers embedded to each $E R$ damper.

- Both methods depend on a small number of variables, so the cost of their implementation is actually very low, being able to run online in real-time. While the $L P V$ observer resides in the combination of two 
simple linear models, the sliding-mode one computes a sign function to induce sliding motion; note that there is no need for online optimization procedures.

Both FE schemes could serve for future FTC purposes of $S A$ suspensions systems with $E R$ dampers, but the $L P V$ observer would certainly yield better results, being able to preserve the system stability or some performance specifications, despite the presence of faults.

\section{REFERENCES}

Blanke, M., Izadi-Zamanabadi, R., Bøgh, S.A., and Lunau, C.P. (1997). Fault-tolerant control systems: a holistic view. Control Engineering Practice, 5(5), 693-702.

Chen, L., Patton, R., and Goupil, P. (2016). Application of model-based LPV actuator fault estimation for an industrial benchmark. Control Engineering Practice, 56, 60-74.

Doumiati, M., Martinez, J., Sename, O., Dugard, L., and Lechner, D. (2017). Road profile estimation using an adaptive youla-kučera parametric observer: Comparison to real profilers. Control Engineering Practice, 61, 270278.

Drakunov, S. and Utkin, V. (1995). Sliding mode observers. tutorial. In Decision and Control, 1995., Proceedings of the 34th IEEE Conference on, volume 4, 3376-3378. IEEE.

Edwards, C., Spurgeon, S.K., and Patton, R.J. (2000). Sliding mode observers for fault detection and isolation. Automatica, 36(4), 541-553.

Fergani, S., Menhour, L., Sename, O., Dugard, L., and D'Andréa-Novel, B. (2016). Integrated vehicle control through the coordination of longitudinal/lateral and vertical dynamics controllers: Flatness and LPV $/ h_{\infty}-$ based design. International Journal of Robust and Nonlinear Control.

Gao, Z. and Ding, S.X. (2007). Actuator fault robust estimation and fault-tolerant control for a class of nonlinear descriptor systems. Automatica, 43(5), 912-920.

Gatzke, E.P. and Doyle Iii, F.J. (2002). Use of multiple models and qualitative knowledge for on-line moving horizon disturbance estimation and fault diagnosis. Journal of Process Control, 12(2), 339-352.

Hamayun, M.T., Edwards, C., and Alwi, H. (2016). Design and analysis of an integral sliding mode fault tolerant control scheme. In Fault Tolerant Control Schemes Using Integral Sliding Modes, 39-61. Springer.

Hernández-Alcántara, D., Tudón-Martínez, J.C., Amézquita-Brooks, L., Vivas-López, C.A., and Morales-Menéndez, R. (2016). Modeling, diagnosis and estimation of actuator faults in vehicle suspensions. Control Engineering Practice, 49, 173-186.

Jiang, J. and Yu, X. (2012). Fault-tolerant control systems: A comparative study between active and passive approaches. Annual Reviews in control, 36(1), 60-72.

Morato, M.M., Sename, O., Dugard, L., and Nguyen, M.Q. (2019). Fault estimation for automotive electrorheological dampers: LPV-based observer approach. Control Engineering Practice, 85, 11-22.

Moreno, J.A. and Osorio, M. (2008). A lyapunov approach to second-order sliding mode controllers and observers. In Decision and Control, 2008. CDC 2008. 47th IEEE Conference on, 2856-2861. IEEE.
Nguyen, M.Q., Sename, O., and Dugard, L. (2016). Comparison of observer approaches for actuator fault estimation in semi-active suspension systems. In 3rd Conference on Control and Fault-Tolerant Systems, 227-232. IEEE.

Poussot-Vassal, C., Sename, O., Dugard, L., Gaspar, P., Szabo, Z., and Bokor, J. (2011). Attitude and handling improvements through gain-scheduled suspensions and brakes control. Control Engineering Practice, 19(3), 252-263.

Poussot-Vassal, C., Spelta, C., Sename, O., Savaresi, S.M., and Dugard, L. (2012). Survey and performance evaluation on some automotive semi-active suspension control methods: A comparative study on a single-corner model. Annual Reviews in Control, 36(1), 148-160.

Rodrigues, M., Hamdi, H., Theilliol, D., Mechmeche, C., and BenHadj Braiek, N. (2015). Actuator fault estimation based adaptive polytopic observer for a class of LPV descriptor systems. International Journal of Robust and Nonlinear Control, 25(5), 673-688.

Rotondo, D., López-Estrada, F.R., Nejjari, F., Ponsart, J.C., Theilliol, D., and Puig, V. (2016). Actuator multiplicative fault estimation in discrete-time LPV systems using switched observers. Journal of the Franklin Institute, 353(13), 3176-3191.

Savaresi, S.M., Poussot-Vassal, C., Spelta, C., Sename, O., and Dugard, L. (2010). Semi-active suspension control design for vehicles. Elsevier.

Sename, O., Tudón-Martínez, J.C., and Fergani, S. (2013). LPV methods for fault-tolerant vehicle dynamic control. In Conference on Control and Fault-Tolerant Systems, 116-130.

Tudón-Martínez, J.C., Varrier, S., Sename, O., MoralesMenendez, R., Martinez, J.J., and Dugard, L. (2013). Fault tolerant strategy for semi-active suspensions with LPV accommodation. In Conference on Control and Fault-Tolerant Systems, 631-636.

Tudón-Martínez, J.C., Fergani, S., Sename, O., Martinez, J.J., Morales-Menendez, R., and Dugard, L. (2015). Adaptive road profile estimation in semiactive car suspensions. IEEE Transactions on Control Systems Technology, 23(6), 2293-2305.

Xiao, B., Hu, Q., and Zhang, Y. (2012). Adaptive sliding mode fault tolerant attitude tracking control for flexible spacecraft under actuator saturation. IEEE Transactions on Control Systems Technology, 20(6), 1605-1612.

Zhang, K., Jiang, B., and Shi, P. (2012). Observer-based fault estimation and accomodation for dynamic systems, volume 436. Springer. 